\title{
Two Pressure-induced Superconducting Anion Radical Salts Exhibiting Different Spin States at Ambient Pressure
}

\author{
Reizo Kato, Akiko Tajima, Akiko Nakao, and Masafumi Tamura \\ RIKEN; JST-CREST, 2-1, Hirosawa, Wako-shi, Saitama, 351-0198, Japan.
}

\section{Supporting Information}

Low-temperature Crystal Structure of EtMe $Z\left[P\left[\operatorname{Pd}(d m i t)_{2}\right]_{2}(Z=P, A s)\right.$

A Weisenberg-type imaging plate system (DIP 320S, Mac Science Co., Inc) equipped with a closed-cycle helium refrigerator (V202C5LZR, DAIKIN Co.) was used for data collection. The structures were solved by the direct method, and refined with the full-matrix least-squared method (SHELXL-97). ${ }^{1}$ All calculations were performed using teXsan crystallographic software package of the Molecular Structure Co.

1) G. M. Sheldrick, SHELXL97, Program for the Refinement of Crystal Structures. University of Gottingen, Germany (1997).

The $10 \mathrm{~K}$ structure of $\mathrm{EtMe}_{3} \mathrm{P}\left[\mathrm{Pd}(\mathrm{dmit})_{2}\right]_{2}$ contains two crystallographically independent anions (blue and yellow molecules in Figure S1) that form a dimer, while in the room temperature structure every dimer is centrosymmetric and the number of crystallographically independent anion is one. All the dimers are equivalent. Distances between best molecular planes, each of which is defined by Pd and four central S atoms, indicate alternation of the inter-dimer distances (Figure S1). The alternation of the inter-dimer distances is associated with that of the inter-dimer HOMO-HOMO overlap integral $B\left(-3.62 \times 10^{-3},-2.99 \times 10^{-3}\right)$.

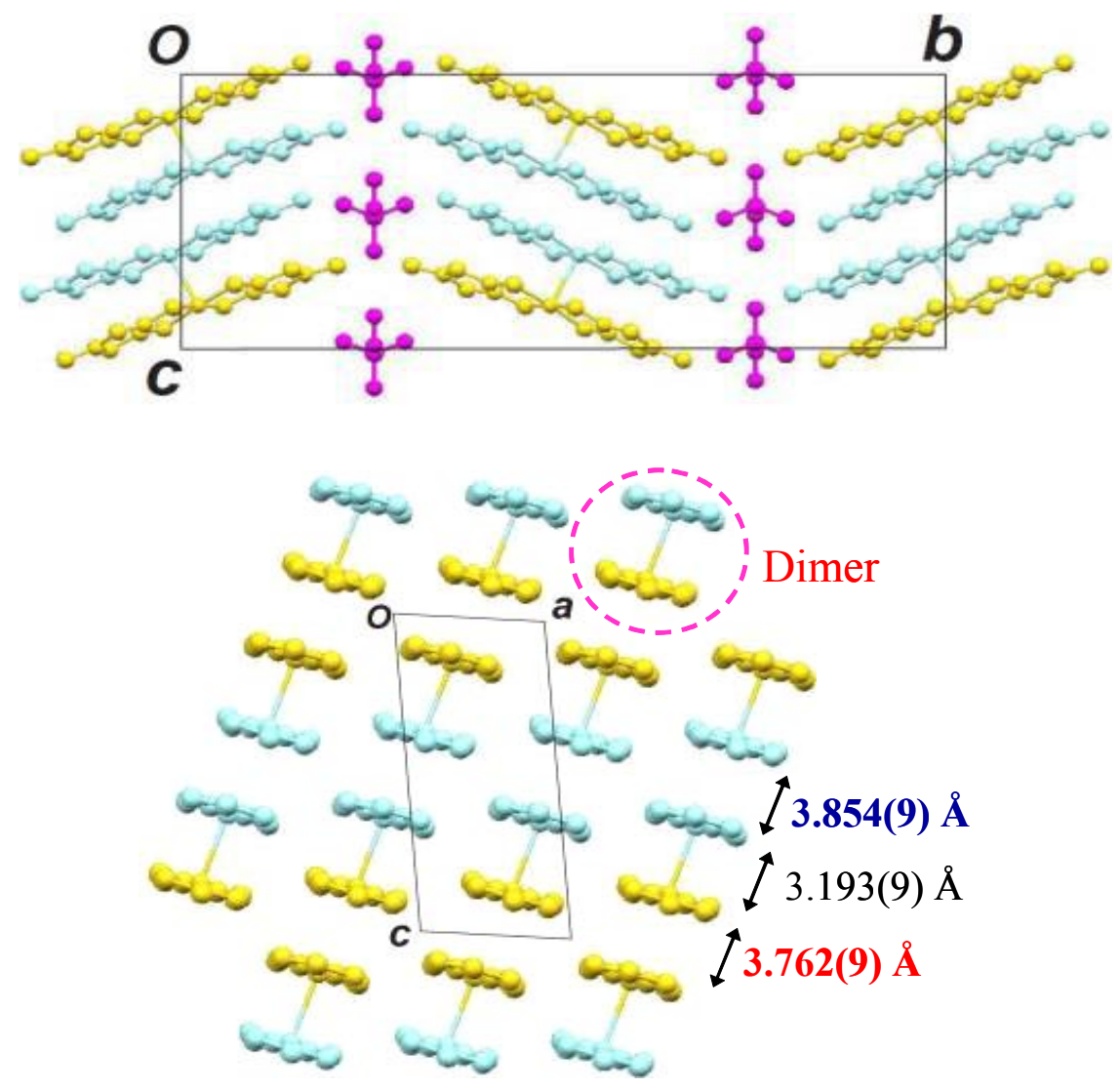

Figure S1. Crystal structure and dimer arrangement in $\mathrm{EtMe}_{3} \mathrm{P}\left[\mathrm{Pd}(\mathrm{dmit})_{2}\right]_{2}$ at $10 \mathrm{~K}$. 
The room temperature structure of $\mathrm{EtMe}_{3} \mathrm{As}\left[\mathrm{Pd}(\mathrm{dmit})_{2}\right]_{2}$ has a monoclinic lattice with the space group $C 2 / \mathrm{c}$. The cation is on a 2-fold axis and thus there are two possible positions for the ethyl group, which leads to the orientational disorder of the cation (Figure S2).

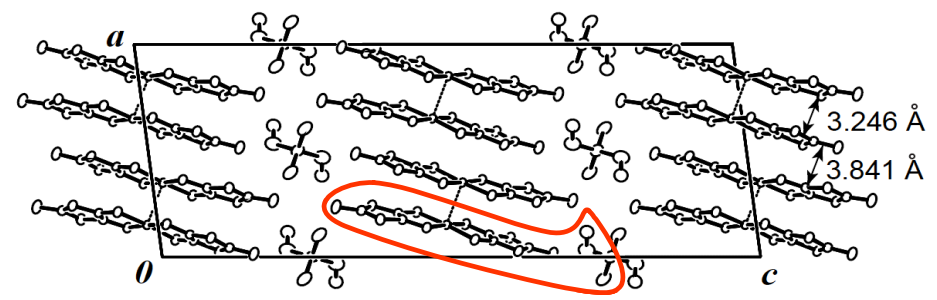

asymmetric unit

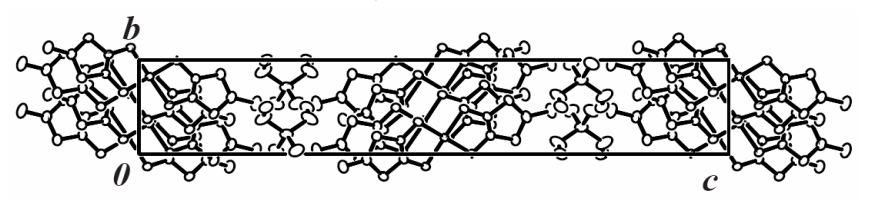

Figure S2. Crystal structure of $\mathrm{EtMe}_{3} \mathrm{As}\left[\mathrm{Pd}(\mathrm{dmit})_{2}\right]_{2}$ at room temperature.

At $150 \mathrm{~K}$, every examined crystal was composed of twinning domains. Each domain has a triclinic lattice with the space group P1 and the lattice vectors of $\boldsymbol{a}_{\mathrm{t}}=-\boldsymbol{b}_{\mathrm{m}}, \boldsymbol{b}_{\mathrm{t}}=\left(\boldsymbol{a}_{\mathrm{m}}+\boldsymbol{b}_{\mathrm{m}}\right) / 2, \boldsymbol{c}_{\mathrm{t}}=\boldsymbol{c}_{\mathrm{m}}$ and $\boldsymbol{a}_{\mathrm{t}}^{\prime}=-\boldsymbol{b}_{\mathrm{m}}, \boldsymbol{b}_{\mathrm{t}}^{\prime}=\left(-\boldsymbol{a}_{\mathrm{m}}+\boldsymbol{b}_{\mathrm{m}}\right) / 2, \boldsymbol{c}_{\mathrm{t}}^{\prime}=-\boldsymbol{c}_{\mathrm{m}}$, where $\boldsymbol{a}_{\mathrm{m}}, \boldsymbol{b}_{\mathrm{m}}$, and $\boldsymbol{c}_{\mathrm{m}}$ are those of the monoclinic structure (Figure S3). The volume ratio of the twin components depends on the crystal. Data collection was carried out for each triclinic domain and it has been confirmed that they have the same structure.
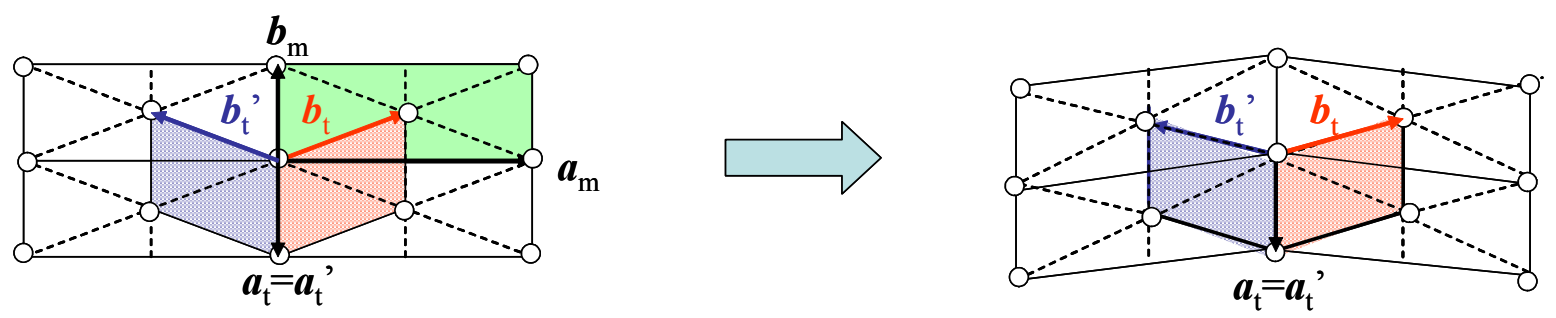

Figure S3. Relation between the crystal axes of the room temperature structure and those of the triclinic structures of twinning domains for $\mathrm{EtMe}_{3} \mathrm{As}\left[\mathrm{Pd}(\mathrm{dmit})_{2}\right]_{2}$.

The triclinic structure contains two crystallographically independent cations and the disorder is removed (Figure S4). Two conduction layers become non-equivalent (indicated by red and black colors in Figure S4).
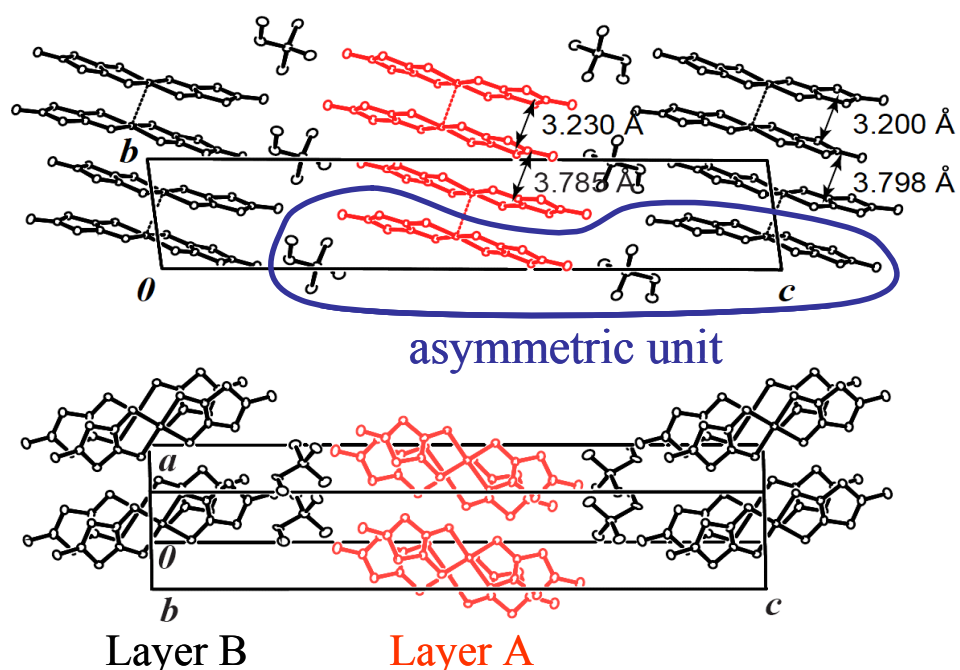

Figure S4. Crystal structure of $\mathrm{EtMe}_{3} \mathrm{As}\left[\mathrm{Pd}(\mathrm{dmit})_{2}\right]_{2}$ at $150 \mathrm{~K}$. 
Table S1 shows difference in intra- and inter-dimer HOMO-HOMO overlap integrals between Layers A and B, which indicates that anisotropy of the inter-dimer interactions is not affected by the transition.

Table S1. Intra- and inter-dimer HOMO-HOMO overlap integrals for $\mathrm{EtMe}_{3} \mathrm{As}\left[\mathrm{Pd}(\mathrm{dmit})_{2}\right]_{2}$ at $150 \mathrm{~K}$.

\begin{tabular}{lll}
\hline & Layer A & Layer B \\
\hline$A$ & -46.72 & -45.41 \\
$B$ & -3.58 & -3.21 \\
$r$ & -2.63 & -2.40 \\
$s$ & -3.50 & -3.22 \\
\hline
\end{tabular}

\section{Magnetic susceptibility of EtMe $\mathrm{E}_{3} \mathrm{Z}\left[\mathrm{Pd}(\mathrm{dmit})_{2}\right]_{2}(\mathrm{Z}=\mathrm{P}, \mathrm{As})$}

Magnetic susceptibility $\chi$ of the randomly oriented polycrystalline samples was measured by use of a Quantum Design MPMS XL7 magnetometer. The magnetization varied almost linearly with field up to $7 \mathrm{~T}$, above $15 \mathrm{~K}$ for $\mathbf{1}$, or above $23 \mathrm{~K}$ for 2 . The data, collected over $5-290 \mathrm{~K}$ at $5 \mathrm{~T}$, were corrected for the diamagnetic contribution ${ }^{2}$ and for the Curie-like contribution from the crystal imperfections.

2) M. Tamura and R. Kato, J. Phys.: Condens. Matter., 2002, 14, L729.

Figure S5 shows the temperature dependence of $\chi$ for $\mathrm{EtMe}_{3} \mathrm{P}\left[\mathrm{Pd}(\mathrm{dmit})_{2}\right]_{2}$, together with the calculated $\chi$ for the triangular lattice model with $J / k_{\mathrm{B}}=250 \mathrm{~K} \cdot \chi$ shows no hysteresis over the whole temperature range. The measurements for a mosaic of oriented crystals indicate that the $\chi(T)$ behavior is magnetically isotropic.

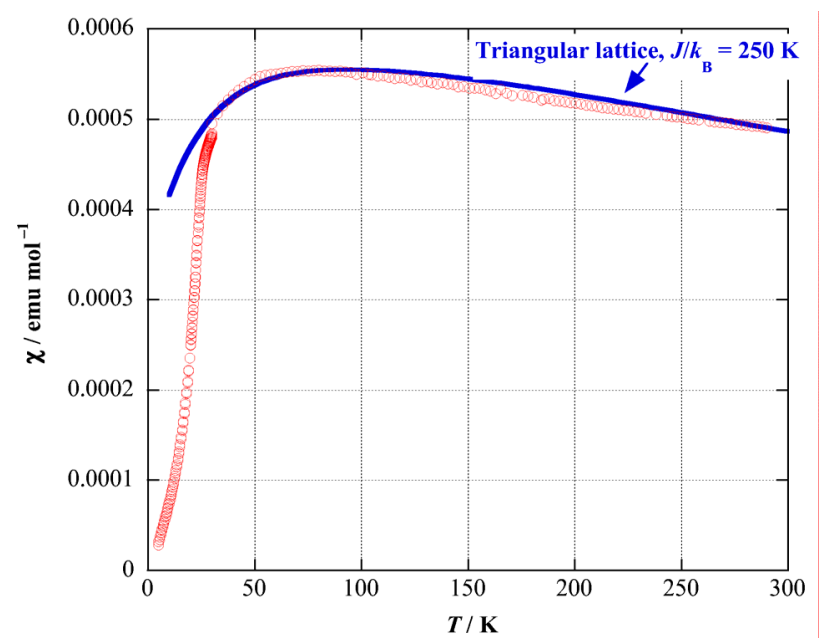

Figure S5. Temperature dependence of magnetic susceptibility for $\mathrm{EtMe}_{3} \mathrm{P}\left[\mathrm{Pd}(\mathrm{dmit})_{2}\right]_{2}$.

Figure S6 shows the temperature dependence of $\chi$ for $\operatorname{EtMe}_{3} \mathrm{As}\left[\mathrm{Pd}(\mathrm{dmit})_{2}\right]_{2}$, together with the calculated $\chi$ for the triangular lattice model with $J / k_{\mathrm{B}}=260 \mathrm{~K}$.

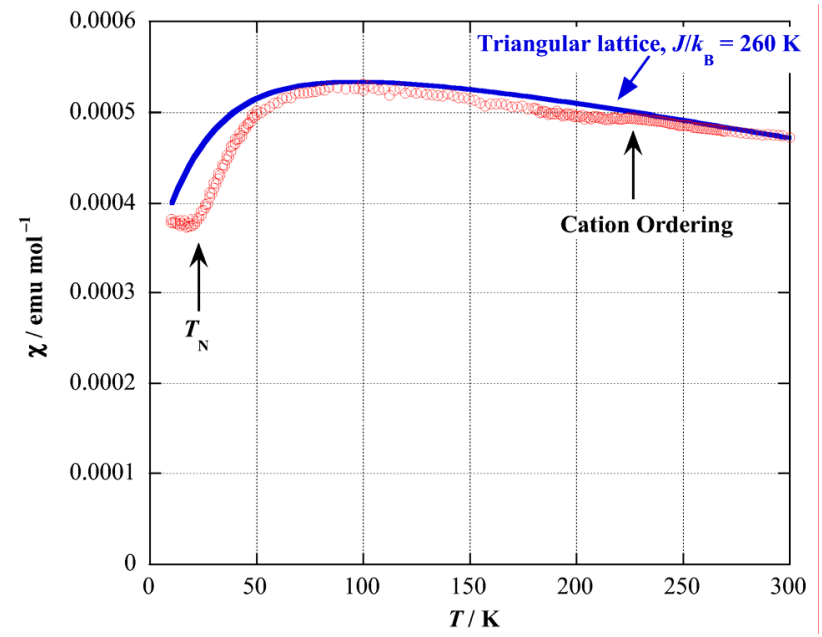

Figure S6. Temperature dependence of magnetic susceptibility for $\mathrm{EtMe}_{3} \mathrm{As}\left[\mathrm{Pd}(\mathrm{dmit})_{2}\right]_{2}$. 
The $\chi$ measurements for aligned crystals at $1 \mathrm{~T}$ indicate anisotropic behavior of $\chi$ below the Néel temperature $T_{\mathrm{N}}$, which indicates that an easy axis is perpendicular to the conduction plane (Figure S7).

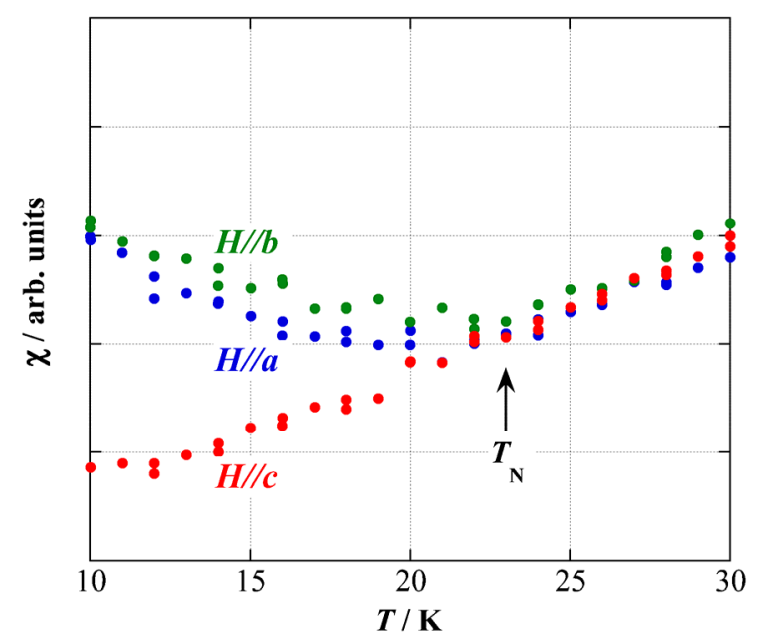

Figure S7. Anisotropy of magnetic susceptibility for $\mathrm{EtMe}_{3} \mathrm{As}\left[\mathrm{Pd}(\mathrm{dmit})_{2}\right]_{2}$. 